\title{
SINKING SKIN FLAP SYNDROME AND PARADOXICAL HERNIATION PROVOKED BY A MALFUNCTION OF EXTERNAL LUMBAR DRAINAGE AND CSF LEAK
}

\author{
Hanko M, Snopko P, OpsenaK R, Benco M, Richterova R, Kolarovszki B. \\ Clinic of Neurosurgery, Jessenius Faculty of Medicine in Martin, Comenius University in \\ Bratislava, University Hospital Martin, Slovakia
}

\begin{abstract}
A b s tract
The sinking skin flap syndrome represents a less-frequent complication in patients after a decompressive craniectomy. It is defined as a neurological deterioration accompanied by a flat or concave deformity of the craniectomy-related skin flap. The underlying brain parenchyma is distorted correspondingly with its blood flow and metabolism being impaired and cerebrospinal fluid hydrodynamics being disturbed, thus causing cerebral dysfunction and neurological symptomatology. The most important options for reversal of this syndrome include Trendelenburg position, maintaining of the cerebrospinal fluid balance, and cranioplasty as a definite solution. We present a patient who underwent a decompressive craniectomy complicated by a cerebrospinal fluid leak in the operative wound treated by means of an external lumbar drainage. Subsequently he developed the sinking skin flap syndrome and a paradoxical cerebral herniation after the drainage system malfunction with a massive cerebrospinal fluid leak at the site of the lumbar drain insertion parallel to the drain itself. His symptoms were, however, successfully alleviated by a positional change, rehydration, and interruption of the lumbar drainage. This illustrational case suggests that clinicians should be aware that patients after decompressive craniectomy may develop a sinking skin flap syndrome as it may either represent an acute risk of a paradoxical brain herniation or complicate the further postoperative care if developed in a chronic way.
\end{abstract}

Keywords: decompressive craniectomy, sinking skin flap syndrome, syndrome of the trephined, paradoxical herniation

\section{INTRODUCTION}

As an effective surgical method designed to control a pathologically elevated intracranial pressure (ICP) triggered typically by malignant middle cerebral artery infarction, traumatic brain injury, intracerebral haemorrhage, inflammatory cerebral affections, or by other lessfrequent causes, decompressive craniectomy (DC) - a surgical removal of cranial bone flap and opening of underlying dura mater - has been used in neurosurgical practice for decades [1]. However effective this surgery may be, only a limited number of patients achieves a favourable outcome as well. In the last report from our clinic concerning the DC for traumatic brain injury we observed a good outcome in $29.17 \%$, mortality in $29.17 \%$, and survival with a severe disability in $41.67 \%$ of our patients [2]. Amongst the factors contributing to a cerebral dysfunction following the DC there is the very principle of this surgery: conversion of the previously closed cranial vault to an "open-box" [3] which may ini-

Corresponding author: Branislav Kolarovszki; e-mail: kolarovszki@jfmed.uniba.sk; phone: +421 434203903 (c) 2019 Kolarovszki B. et al.

This work is licensed under the Creative Commons Attribution-NonCommercial-NoDerivs 4.0 License (https://creativecommons.org/licenses/by-nc-nd/4.0/) 
tially reduce the ICP but also complicate the relationship between brain metabolism, perfusion, atmospheric pressure, and cerebrospinal fluid (CSF) dynamics in the decompressed area $[3,4,5,6]$, thus causing various neurological symptomatology. With an exclusion of other intra- and extracranial causes a term "syndrome of the trephined" (SOT) was introduced for a wide spectrum of neurological symptoms in patients with cranial defects which are typically reversible after a cranioplastic surgery [7]. On the other hand, the "sinking skin flap syndrome" (SSFS) is described as a neurological deterioration with a variably delayed onset after the DC which presents with a subsidence of the skin flap under the level of the adjacent cranial surface [8] accompanied by a compression of the intracranial contents and their shift to the contralateral site, often with an impeding paradoxical brain herniation $[3,9]$. Hereby we report a case of a patient who underwent the DC after an evacuation of an atypical intracerebral haematoma at our institution and later developed the SSFS and a paradoxical cerebral herniation provoked by a malfunction of an external lumbar drainage system with a CSF leak at the site of the lumbar catheter insertion parallel to the catheter itself.

\section{CASE PRESENTATION}

A 60-year-old male with a history of an inoperable oesophageal carcinoma treated by chemotherapy and external radiotherapy and with a history of a long-term warfarin intake due to a paroxysmal atrial fibrillation was admitted to our clinic form a peripheral hospital with a diagnosis of a spontaneous atypical intracerebral haematoma (Fig 1) predominantly located in the right temporal lobe extending parietally and into the ventricular system. He presented with a GCS (Glasgow Coma Scale) value of 9 and with a severe left-sided hemiparesis, he was isocoric with pupillary reflex bilaterally preserved. Evacuation of the haematoma was indicated and due to presence of an intraoperative brain oedema a DC was also performed. As a material for augmentative duraplasty a fascia lata graft was used and fixed by interrupted sutures, a watertight dural suture was not performed, and an ICP sensor was not implanted.

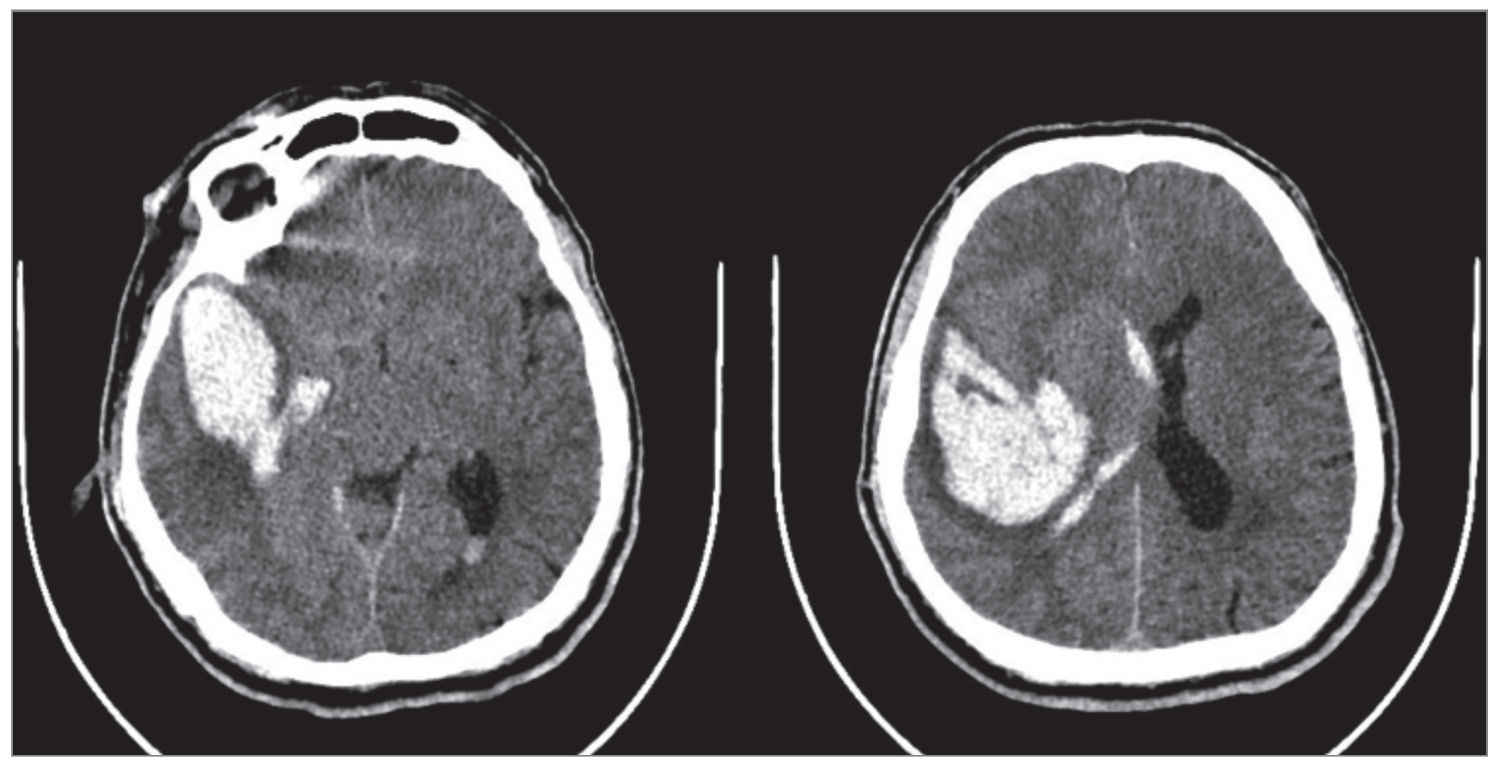

Fig. 1 Preoperative CT scan showing an intracerebral haemorrhage in the right temporal lobe extending into the ventricular system 
After repeated CT scans showing adequate postoperative findings with a reduction of the midline shift (Fig 2), the patient was transferred back to the peripheral hospital on the $5^{\text {th }}$ postoperative day and a tracheostomy was performed here.

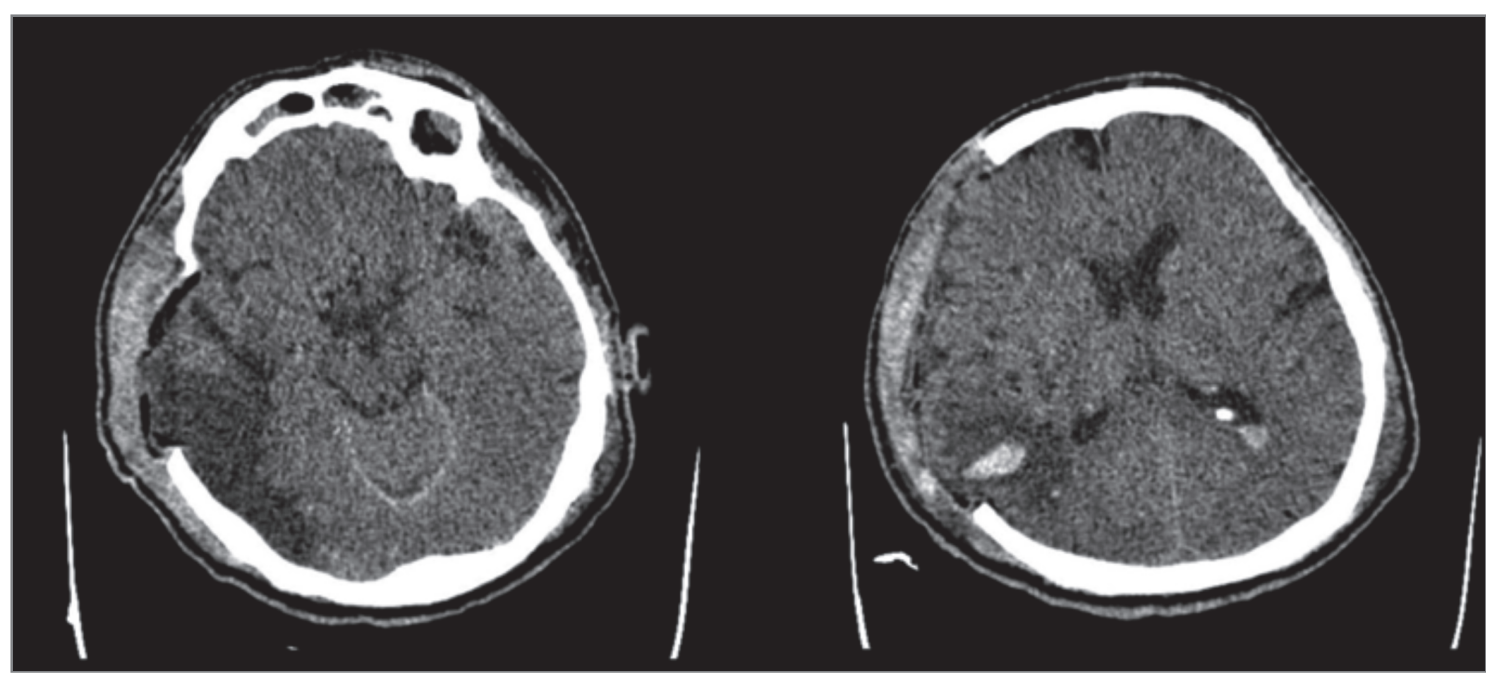

Fig 2 Postoperative CT control displaying an adequate decompression acquired by means of the haematoma evacuation and decompressive craniectomy. The midline shift is resolved, there is a residual haemocephalus and a region of haemorrhagic infarction at the posterior site of the cranial defect

On the $13^{\text {th }}$ postoperative day the patient was readmitted to our hospital because of the operative wound dehiscence with a CSF leak in the posterior part of the question-mark skin incision. The sedation was already discontinued and the patient was conscious (GCS 14), communicating by gestures and even verbally, obeying simple commands. A residual leftsided severe hemiparesis was present. A resuture of the leaking site of the operative wound was performed and, in order to alleviate the CSF pressure, an external lumbar drainage (ELD) system was placed in the L4/5 intervertebral space. No further CSF leak in the operative wound was observed, however after six days a malfunction of the ELD occurred possibly due to its partial extraction during the patient's movement. A new ELD system was placed through the same entry-point. 18 hours after the ELD reinsertion the patient started complaining of a severe headache and his condition rapidly deteriorated down to GCS 8 with no spontaneous limb movement but localising pain, no verbal response, and eye-opening to a painful stimuli only. His heart rate was increased up to 110 beats per minute. A massive CSF leak was observed at the site of the ELD insertion parallel to the lumbar drain itself accompanied by a newly apparent concave deformity of the craniectomy skin flap. An emergent CT control displayed a concave compression of the brain tissue in the area of the DC, compression and displacement of the ventricular system, and a midline shift of $10 \mathrm{~mm}$ to the contralateral site with a presence of a paradoxical brain herniation (Fig 3).

As a diagnosis of SSFS was made the ELD was temporarily interrupted, the patient was placed into the Trendelenburg position and intravenously rehydrated. The CSF leak did not continue at the moment most likely because of the large CSF volume being already lost. After the described countermeasures were performed the patient's neurological status gradually improved in a matter of hours with patient being conscious, spontaneously moving his rightsided extremities and communicating again, reporting a relief of the cephalea. The skin flap sinking also resolved. After 24 hours we continued in the ELD regime until the $13^{\text {th }}$ day when it was removed due to another (however minor) CSF leak at the site of its insertion. At that time the operative wound was well healed with no signs of dehiscence or CSF fistula. Since 


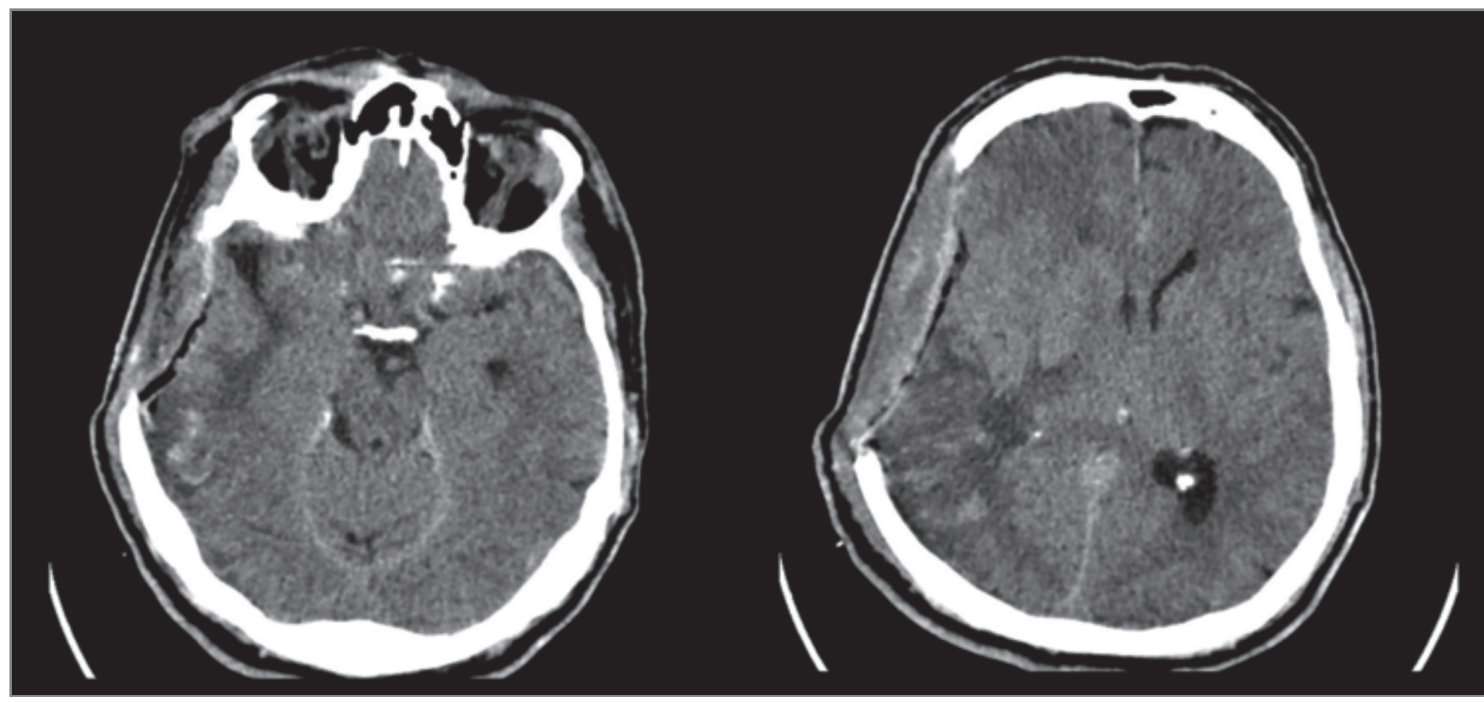

Fig. 3 CT findings following the CSF leak. Concave brain deformity at the site of removed bone flap, compression of ventricular system, and paradoxical midline shift to the left side (10mm measured) are clearly visible.

the patient's condition was complicated by a severe aspiration pneumonia and leukopenia we did not consider him for an early cranioplasty. Also due to a presence of a suspected metastatic focus (which was however not confirmed) the bone flap was not preserved. It was, however, possible to perform the cranial reconstruction (Fig. 4) in a delayed manner - 15 months later with use of an individualised 3D printed titanium alloy implant (Biomedical Engineering, Košice, Slovakia). No serious complications were encountered. The patient's clinical status was considerably improved even prior to the cranioplastic surgery as the leftsided hemiparesis was already alleviated to a subtle level only with patient being able to walk and communicate independently, scoring GOS (Glasgow Outcome Score) 4 and 2 on the Modified Rankin Scale and this neurological picture remained stable.

Fig. 4 A lateral X-ray image displaying the extent of cranial reconstruction using a custom-made 3D printed titanium alloy implant (Biomedical Engineering, Košice, Slovakia)

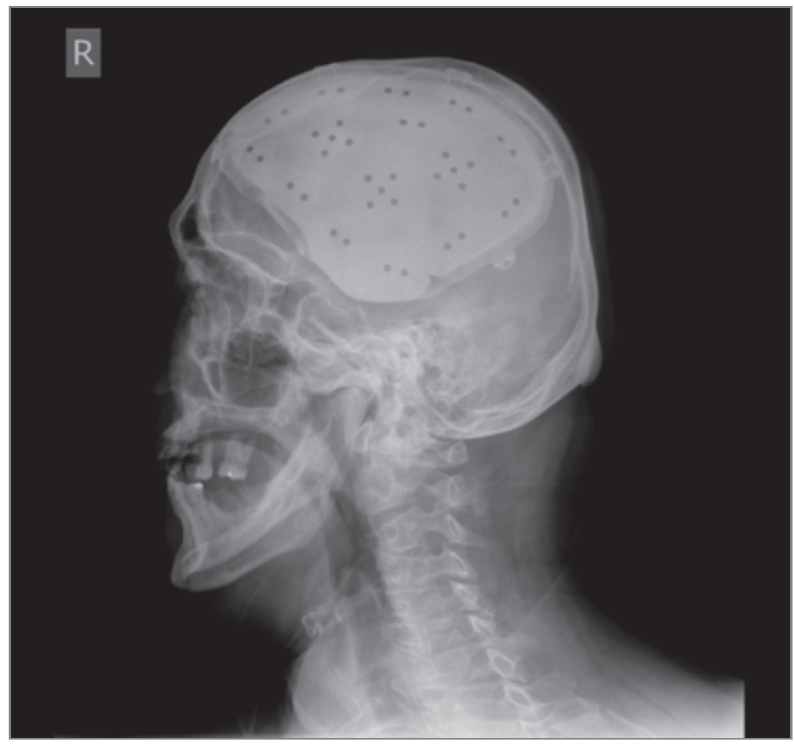




\section{DISCUSSION}

A cranial bone defect resulting from the DC does not only represent a cosmetic disorder but as its very presence changes the pathophysiologic relationships between the intracranial and extracranial space, it may be accompanied by a further neurological deficit which is, however, often reversible [5,7,8]. In 1939 Grant and Norcross have introduced the term "syndrome of the trephined" as a combination of symptoms including headaches, mental and speech disturbances, sensory and visual changes, fatigue, and dizziness in patients with cranial defects [7]. It has been summarized that in SOT the symptoms usually begin weeks to months after the craniectomy with no relation to the location of the primary intracranial lesion and an improvement was observed after a cranioplastic surgery $[4,7,9,10]$. Later, in 1977 , a neurological deterioration with a presence of a concave deformity of the skin flap above the cranial bone defect in post-DC patients has been reported by Yamaura and Makino and named "sinking skin flap syndrome". The symptoms were typically worsened in an upright position of the patient [11]. Compression of the brain parenchyma by an effect of the atmospheric pressure was provided as an explanation of this phenomenon [8]. A rapid improvement of patients' status was also observed after the cranioplasty, but the authors strongly distinguished the SSFS from the previously reported SOT which was mostly represented by subjective complaints of the patients [8]. Another clinical entity, the "motor trephine syndrome", was described in patients after DC for traumatic brain injury. It develops with a longer delay (median 4.5 months after craniectomy) and is associated with present postcontusion foci and CSF flow disturbances after DC (subdural hygromas and hydrocephalus). Clinical picture shows a reversible monoparesis contralateral to the side of DC that is quickly reversible by cranioplasty. CT and perfusion studies reveal an intraparenchymal CSF transgression into the former contusion foci with further oedema formation and subsequent local perfusion attenuation in these regions. Cranial reconstruction improves these findings as well [12,13]. It is, however, undeniable that all the above mentioned clinical entities are not always clearly differentiated even in current neurosurgical literature $[9,14]$.

In patients with the SSFS the typical symptoms include alteration of consciousness, headache, cranial nerve palsies, worsening of the limb-paresis, and speech dysfunction. Dysautonomic syndrome presenting with paroxysms of increased blood pressure, heart and respiratory rate, and profound swelling has also been reported $[8,11,15,16]$.

Numerous causes of the SSFS have been described, often linked to the size of the skull defect and presence of a pressure gradient between the extracranial and intracranial space [3]. A typical subacute or chronical onset of SSFS is associated with a large DC with no other provoking factors being present except a positional change and dehydration $[17,18,19,20]$. SSFS with a more acute onset may occur in situations with a present CSF leak or diversion such as in patient with an implanted ventriculo-peritoneal shunt $[6,11,16,21,22,23,24]$ or external lumbar drainage [25]. If the volume of intracranial contents is swiftly lowered, such as during hyperventilation or after administration of osmotic diuretics, the SSFS can also develop [1,3].

CT findings in patients with the SSFS typically display the concave distortion of the brain parenchyma corresponding to the cranial bone defect, a compression of the ipsilateral ventricle, and a contralateral shift of the midline structures including the third ventricle. There may also be signs of an imminent paradoxical brain herniation [3,9,11].

Treatment modalities for reversing of the SSFS with an acute onset include restoration of the balanced CSF hydrodynamics by moving the patient into Trendelenburg position and restoring the CSF volume by means of adequate rehydration, setting a higher opening pressure of ventriculo-peritoneal shunt valves or even a temporary ligation of the shunt catheter, and the ELD cancellation with a possible use of a lumbar blood patch $[11,16,22,25]$. The traditional countermeasures against the intracranial hypertension (such as diuretics and 
osmotherapy) during a present SSFS, however, pose a serious risk of patient's status worsening and may lead to the paradoxical brain herniation [3,20]. Due to the conservative treatment of the SSFS being not always effective an early cranial defect reconstruction was proven to be a definite solution for the SSFS as it turns the intracranial space to its original "closed-box" state, thus alleviating the disturbed relationships between the intracranial compartments $[3,16,17,18]$.

There is also an alternative surgical method for cranial decompression that avoids the need for subsequent cranioplasty and also avoids the complications associated with the bone flap removal. In carefully indicated cases where a DC would be too radical (for example the intraoperative brain oedema is not too massive or its postoperative development is not expected), an osteoplastic decompressive craniotomy may be performed. During this surgery a large craniotomy with an oblique bone incision using a Gigli saw is performed, but the bone flap remains connected to the temporalis muscle which later acts as a hinge. Durotomy and sanation of an eventual intracranial mass lesion is done and then the augmentative duraplasty is performed. The relatively unfixed bone flap remains elevated during a presence of intracranial tissue expansion and later returns to its original position and fuses with the surrounding bone. If the osteoplastic decompressive craniotomy proves as insufficient in any individual patient, it can always be converted to a standard DC $[1,26]$.

Various models have been introduced in order to explain the pathophysiologic mechanisms responsible for the neurological deficits associated with the SSFS. It was proposed that after the craniectomy the adjacent brain parenchyma unprotected by the bony vault is affected by the atmospheric pressure which is transmitted through the sunken skin flap $[6,8,11]$. This external force causes the concave deformity of the skin with a distortion of the meninges, compression of the subarachnoid space and the underlying brain tissue, thus altering the cerebral blood inflow and venous outflow, cerebral metabolism, and CSF flow velocity and resorption [3,4,8,27,28]. Due to an almost direct transmission of the atmospheric pressure onto the intracranial space the ICP tends to equalize with the atmospheric pressure $\left(1033 \mathrm{~cm}\right.$ of $\left.\mathrm{H}_{2} 0\right)$ and if a rapid change of the intracranial compartment volume occurs, such as during the upright position, dehydration or during a CSF leak the intracranial contents may be pushed downwards through the tentorial notch and foramen magnum leading to an aggravation of present symptoms and even to a paradoxical cerebral herniation $[3,8,10,15,25,28]$. The CSF dynamics is typically restored after the cranioplastic surgery which results in normalisation of the ICP [4,8]. Clinical studies using MR perfusion scans [29], MR spectroscopy [30], CT perfusion [6], xenon-enhanced CT perfusion scans [30,31], and transcranial Doppler-ultrasound [5] have similarly proven a subnormal cerebral blood flow in patients who underwent DC. The cerebral blood flow increase occurred after cranioplastic surgery with symptoms of SSFS and SOT being alleviated [29,30,31]. These cerebral blood flow changes affect not only the hemisphere adjacent to the cranial defect but the contralateral one as well [6,31]. The cerebral glucose metabolism is also increased after cranioplasty, as reported in a PET-CT study performed by Winkler et al. [5]. All reports mentioned therefore confirm a normalisation of intracranial conditions altered by the DC and a therapeutic value of cranioplastic surgery. The suddenly increased cerebral blood flow and volume after cranioplasty can, however, occasionally culminate into a reperfusion injury and a secondary intracerebral haemorrhage probably because of an impaired vascular resistance in the previously damaged hemisphere [32,33].

In the case we hereby present a sudden loss of the CSF volume and pressure due to the leak at the site of the ELD catheter insertion most likely resulted in a rapid increase of pressure gradient between the extracranial, intracranial, and intraspinal space creating the concave scalp deformity typical for SSFS. The patient's brain was pushed to the site contralateral to cranial defect (as proven by CT verified paradoxical herniation with midline shift) by a combination of the atmospheric pressure and gravity force and the meninges were similarly distorted, thus causing the sharp headaches. As the brain shifting progressed the cerebral blood flow was presumably disturbed and the cerebral dysfunction 
resulted in an unresponsive status. This explanation would be correspondent with the findings reported by Yamaura, Nakamura, Yoshida, and Zhao [8,11,25,30]. After a proper management by means of the ELD interruption, sufficient rehydration, and Trendelenburg position the patient's status gradually improved and the skin flap deformity disappeared most likely because of restoration of the CSF volume and the pressure gradient reversal caused by Trendelenburg position. This example demonstrates a possibility to reverse a SSFS and the paradoxical brain herniation with the acute onset and highlights a need of quick recognition of this condition in order to provide adequate countermeasures.

\section{CONCLUSION}

The SSFS represents a less-frequent complication of DC that can, however, cause longterm neurological dysfunction or an acute and more serious worsening of the patient's status and even lead to the paradoxical cerebral herniation. It should be, therefore, recognized as fast as possible by the clinicians. The treatment is based on the restoration of CSF hydrodynamics with the early cranioplasty being the ultimate solution.

\section{REFERENCES}

1. Mraček J. Dekompresivní kraniektomie. Praha: Galén; 2016.

2. Hanko M, Richterová R, Kolarovszki B. Efficiency and Limitations of Decompressive Craniectomy in Patients after Traumatic Brain Injury - Preliminary Results. Acta Medica Martiniana 2017; 17(3):32-38.

3. Akins PT, Guppy KH. Sinking Skin Flaps, Paradoxical Herniation, and External Brain Tamponade: A Review of Decompressive Craniectomy Management. Neurocrit Care 2008; 9(2):267-276.

4. Dujovny M et al. Cranioplasty: Cosmetic or Therapeutic? Surg Neurol 1997; 47(3):238-241.

5 . Winkler PA et al. Influence of cranioplasty on postural blood flow regulation, cerebrovascular reserve capacity, and glucose metabolism. J Neurosurg 2000; 93(1):53-61.

6. Sakamoto S et al. CT perfusion imaging in the syndrome of the sinking skin flap before and after cranioplasty. Clin Neurol Neurosurg 2006; 108(6):583-585.

7. Grant FC, Norcross NC. Repair of Cranial Defects by Cranioplasty. Ann Surg 1939; 110(4):488512.

8. Yamaura A, Makino H. Neurological deficits in the presence of the sinking skin flap following decompressive craniectomy. Neurol Med Chir (Tokyo) 1977; 17(1):43-53.

9. Vasung L et al. Radiological signs of the syndrome of the trephined. Neuroradiology 2016; 58(6):557-568.

10. Ashayeri K et al. Syndrome of the Trephined: A Systematic Review. Neurosurgery 2016; 79(4):525534.

11. Nakamura T et al. Rapid neurological alteration associated with concave deformity of the skin flap in a craniectomized patient. Case report. Neurol Med Chir (Tokyo) 1980; 20(1):89-93.

12. Stiver SI, Wintermark M, Manley GT. Motor trephine syndrome: A mechanistic hypothesis. Acta Neurochir Suppl 2008; 102:273-277.

13. Stiver SI, Wintermark M, Manley GT. Reversible monoparesis following decompressive hemicraniectomy for traumatic brain injury. J Neurosurg 2008; 109(2):245-254.

14. Annan M et al. Sinking skin flap syndrome (or Syndrome of the trephined): A review. Br J Neurosurg 2015; 29(3):314-318.

15. Honeybul S. Neurological susceptibility to a skull defect. Surg Neurol Int 2014; 5:83.

16. Romero FR et al. Sinking skin flap syndrome with delayed dysautonomic syndrome - An atypical presentation. Int J Surg Case Rep 2013; 4(11):1007-1009.

17. Gulia M, George RA, Anas N. Sinking Skin Flap Syndrome: Post Craniectomy Complication: A Case Report. International Journal Of Advances In Case Reports 2015; 2(18):1110-1113. 
18. Joseph V, Reilly P. Syndrome of the trephined. J Neurosurg 2009; 111(4):650-652.

19. Liew BS, Rosman AK, Adnan JS. Sinking Skin Flap Syndrome. Clin Intensive Care Med. 2017; 2:42-48.

20. Michael AP, Espinosa J. Paradoxical Herniation following Decompressive Craniectomy in the Subacute Setting. Case Rep Neurol Med 2016; 2090384.

21. Ossig C et al. Sinking Skin Flap Syndrome after Hemicraniectomy and Ventriculo-Peritoneal Shunt Overdrainage. J Neurol Neurophysiol 2015; 6:272.

22. Kim SY et al. Sinking Skin Flap Syndrome after Craniectomy in a Patient Who Previously Underwent Ventriculoperitoneal Shunt. Korean J Neurotrauma 2012; 8(2):149-152.

23. Jeyaraj P. Importance of Early Cranioplasty in Reversing the "Syndrome of the Trephine/Motor Trephine Syndrome/Sinking Skin Flap Syndrome”. J Maxillofac Oral Surg 2015; 14(3):666-673.

24. Waranabe J, Maruya J, Nischimaki K. Sinking skin flap syndrome after unilateral cranioplasty and ventriculoperitoneal shunt in a patient with bilateral decompressive craniectomy. Interdiscip Neurosurg 2016; 5:6-8.

25. Zhao $\mathrm{J}$ et al. Sinking skin flap syndrome and paradoxical herniation secondary to lumbar drainage. Clin Neurol Neurosurg 2015; 133:6-10.

26. Mraček J et al. Osteoplastic decompressive craniotomy - an alternative to decompressive craniectomy. Acta Neurochir (Wien) 2011; 153(11):2259-2263.

27. Fodstad $\mathrm{H}$ et al. Effect of cranioplasty on cerebrospinal fluid hydrodynamics in patients with the syndrome of the trephined. Acta Neurochir (Wien) 1984; 70(1-2):21-30.

28. Segal DH, Oppenheim JS, Murovic JA. Neurological Recovery after Cranioplasty. Neurosurgery 1994; 34(4): 729-731.

29. Kemmling A et al. Case report of MR perfusion imaging in Sinking Skin Flap Syndrome: growing evidence for hemodynamic impairment. BMC Neurol 2010; 10:80.

30. Yoshida K et al. Dynamics of cerebral blood flow and metabolism in patients with cranioplasty as evaluated by ${ }^{133} \mathrm{Xe} \mathrm{CT}$ and ${ }^{31} \mathrm{P}$ magnetic resonance spectroscopy. J Neurol Neurosurg Psychiatry 1996; 61(2):166-171.

31. Isago $\mathrm{T}$ et al. Sinking Skin Flap Syndrome. A Case of Improved Cerebral Blood Flow After Cranioplasty. Ann Plast Surg 2004; 53(3):288-292.

32. Han JH, Lee MS. Intracerebral Hemorrhagic Infarction after Cranioplasty in a Patient with Sinking Skin Flap Syndrome. Korean J Neurotrauma 2016; 12(2):152-155.

33. Kwon SM et al. Reperfusion Injury after Autologous Cranioplasty in a Patient with Sinking Skin Flap Syndrome. J Korean Neurosurg Soc 2012; 51(2):117-119.

\section{ABBREVIATIONS:}

CSF - Cerebrospinal fluid

GCS - Glasgow Coma Scale

GOS - Glasgow Outcome Score

DC - Decompressive craniectomy

ELD - External lumbar drainage

ICP - Intracranial pressure

SSFS - Sinking skin flap syndrome

SOT - Syndrome of the trephined

Acknowledgment: This work was supported by the project: The application of PACS (Picture Archiving and Communication System) in the research and development, ITMS 26210120004.

Received: May, 10, 2019

Accepted: September, 10, 2019 